\title{
Changes in expression of serum chemokine CXCL13 and IL-6 after hip replacement, and the relationship with lower limb vein thrombus
}

\author{
ZHIYU GUAN \\ Department of Tramotology and Orthopedics, The First Affiliated Hospital of Guizhou University \\ of Traditional Chinese Medicine, Guiyang, Guizhou 550001, P.R. China
}

Received October 4, 2019 ; Accepted December 2, 2019

DOI: $10.3892 /$ etm.2019.8365

\begin{abstract}
Changes in the expression of serum chemokine CXC ligand 13 (CXCL13) and interleukin-6 (IL-6), and the relationship with lower limb vein thrombus were explored. A total of 128 patients undergoing hip replacement in The First Affiliated Hospital of Guizhou University of Traditional Chinese Medicine from May 2017 to June 2019 were selected, and the patients suffering from lower limb vein thrombus were enrolled as group A and other patients not suffering from it were enrolled as group B. Enzyme-linked immunosorbent assay was employed to determine the levels of serum chemokine CXCL13 and IL-6, and receiver operating characteristic curves of serum chemokine CXCL13 and IL-6 levels in diagnosing restenosis after surgery were drawn. Pearson's correlation coefficient was adopted to analyze the correlation between serum chemokine CXCL13 and IL-6, and the logistic regression analysis to analyze the risk factors affecting hip replacement in patients. The levels of serum CXCL13 and IL-6 in group A were significantly higher than those in group $\mathrm{B}$ (both $\mathrm{P}<0.001$ ). The specificity and sensitivity of serum CXCL13 level in diagnosis of lower limb vein thrombus after hip replacement were 61.76 and $80.00 \%$, respectively, and those of serum IL-6 level in diagnosis were 70.59 and $66.67 \%$, respectively. Serum CXCL13 level was positively correlated with serum IL-6 level $(\mathrm{P}<0.001)$, and age, body mass index (BMI), CXCL13 level and IL-6 level of the patients were independent risk factors affecting the efficacy of hip replacement. Serum CXCL13 level and serum IL-6 level can be used as biological indexes for prediction of early lower limb vein thrombus after hip replacement, and logistic
\end{abstract}

Correspondence to: Dr Zhiyu Guan, Department of Tramotology and Orthopedics, The First Affiliated Hospital of Guizhou University of Traditional Chinese Medicine, 71 Baoshan North Road, Guiyang, Guizhou 550001, P.R. China

E-mail: g4o625@163.com

Key words: hip replacement, chemokine CXC ligand 13, interleukin-6, lower limb vein thrombus regression analysis revealed that the age of the patients, BMI, diabetes history, hyperlipidemia history, hypertension history, CXCL13 level and IL-6 level are independent risk factors affecting the efficacy of hip replacement.

\section{Introduction}

Hip replacement is a large-scale complex orthopedic operation requiring long operation time. It is mainly applicable to some fractures (such as femoral neck fracture), osteoarthritis, bone neoplasms, ankylosis or hip dysplasia, and others. As one of the most effective methods for joint correction, hip replacement is mainly to relieve hip joint pain, correct deformity, restore and to improve hip joint movement function (1-3). It uses prostheses similar to human bone joints made of metal materials with good biocompatibility and mechanical properties to replace joint surfaces damaged by diseases or injuries through surgical methods (4-6). However, hip replacement brings a high risk of lower limb deep venous thrombosis, and lower limb deep venous thrombosis is a common complication, which not only affects the efficacy of hip replacement, but also greatly affects the recovery of patients, and even leads to death under serious situations $(7,8)$.

CXC ligand 13 (CXCL13) is a main factor for recruiting $\mathrm{B}$ cells in neuro-inflammatory reaction. In various inflammatory diseases, a large number of monocytes aggregate, and chemokine CXCL13 is expressed in a large amount locally, further leading to inflammatory cascade reaction and local tissue or organ damage (9-11). IL-6, as a very important part of interleukins, and participates in many inflammatory reactions and diseases in human body $(12,13)$. CXCL13 and IL-6 are both important pro-inflammatory factors $(10,13)$. Research shows that the coagulation promoting effect of inflammation and vicious circle caused by the interaction of coagulationinflammation network have become important pathogenesis of thrombosis $(14,15)$.

Therefore, this study compared the patients suffering from lower limb vein thrombus after hip replacement to those not suffering from it after hip replacement in terms of serum CXCL13 and IL-6 levels, and the serum CXCL13 and IL-6 levels were considered as biological indexes for early prediction of lower limb vein thrombus, so as to provide reference 
in clinical practice. This study also analyzed independent risk factors affecting hip replacement, so as to provide reference for protection to patients undergoing hip replacement.

\section{Patients and methods}

Clinical data of patients. A total of 128 patients undergoing hip replacement in The First Affiliated Hospital of Guizhou University of Traditional Chinese Medicine (Guiyang, China) from May 2016 to May 2018, were divided into group A ( $\mathrm{n}=68$ ) and group B $(n=60)$. Group A consisted of 35 males and 33 females, with an average age of $50.7 \pm 7.5$ years, and group B consisted of 35 males and 25 females, with an average age of $50.1 \pm 6.8$ years. There were no statistical differences in sex and age between the two groups (both $\mathrm{P}>0.05$ ).

The study was approved by the Medical Ethics Committee of the Hospital and each patient signed an informed consent form after understanding the requirements of the study.

Inclusion and exclusion criteria. The inclusion criteria were as follows: Patients confirmed with osteoarthritis based on imaging who received hip replacement, patients meeting the diagnostic criteria of Guidance for the Diagnosis and Treatment of Osteoarthritis (2018) (16), patients with all the required clinical data, and patients willing to cooperate with the treatment and follow-up.

Exclusion criteria were as follows: Patients with congenital immunodeficiency, patients with severe infectious diseases or inflammatory disease, patients with other malignant tumors, patients with hepatic or kidney function obstacle, patients with expected survival time less than 3 months, and pregnant or lactating women.

Instruments and reagents. Human IL-6 ELISA kit and human CXCL13 ELISA kit (Shanghai Enzyme-linked Biotechnology Co., Ltd., PI330 and m1057504); blood analyzer (Japan SYSMEX, XS-800i), and supporting reagents for testing blood routine indexes; enzyme-linked immuno-sorbent assay (ELISA) test kit (Wuhan Elabscience Biotechnology Co., Ltd., E-EL-H0102c).

Sample collection and ELISA detection methods. Venous blood $(5 \mathrm{ml})$ was sampled from patients in group A and B, allowed to stand for $30 \mathrm{~min}$, and centrifuged at 1,000-2,000 x g and $4^{\circ} \mathrm{C}$ for $10 \mathrm{~min}$ after the whole blood was naturally coagulated and serum was separated to obtain the supernatant. ELISA was used to detect the expression of serum chemokine CXCL13 and IL-6. A blank well, a standard well and a sample well were set, respectively. Standards with a concentration of 0 were added into the blank well, and $50 \mu$ of standards with different concentrations were added into the standard well. The well for samples to be determined was added with $10 \mu \mathrm{l}$ of the samples, and $40 \mu \mathrm{l}$ of sample diluent. The blank well was not added with sample diluent. Each well was added with $100 \mu 1$ of horseradish peroxidase (HRP)-labeled detection antibody except the blank well. The reaction wells were blocked with a microplate sealer, and incubated in a water bath at $37^{\circ} \mathrm{C}$ for $65 \mathrm{~min}$. The liquid in each well was discarded, and each well was patted to dry with absorbent paper, filled with washing solution, and allowed to stand for $2 \mathrm{~min}$. After
Table I. General data of the patients.

\begin{tabular}{|c|c|c|c|c|}
\hline Variables & $\begin{array}{c}\text { Group A } \\
(\mathrm{n}=68)\end{array}$ & $\begin{array}{c}\text { Group B } \\
(\mathrm{n}=60)\end{array}$ & $\chi^{2}$ & P-value \\
\hline \multicolumn{5}{|l|}{ Sex } \\
\hline Male & $35(51.47)$ & $35(58.33)$ & 0.606 & 0.436 \\
\hline Female & $33(48.53)$ & $25(41.67)$ & & \\
\hline \multicolumn{5}{|l|}{ Age (years) } \\
\hline$<30$ & $28(47.18)$ & $36(60.00)$ & 4.518 & 0.034 \\
\hline$\geq 30$ & $40(58.82)$ & $24(40.00)$ & & \\
\hline \multicolumn{5}{|l|}{ BMI $\left(\mathrm{kg} / \mathrm{m}^{2}\right)$} \\
\hline$\geq 22$ & $41(60.29)$ & $21(35.00)$ & 8.165 & 0.004 \\
\hline$<22$ & $27(39.71)$ & $39(65.00)$ & & \\
\hline \multicolumn{5}{|l|}{ Smoking } \\
\hline Yes & $33(48.53)$ & $26(43.33)$ & 2.170 & 0.141 \\
\hline No & $35(51.47)$ & $34(56.67)$ & & \\
\hline \multicolumn{5}{|l|}{ Drinking } \\
\hline Yes & $38(55.88)$ & $34(56.67)$ & 0.008 & 0.929 \\
\hline No & $30(44.12)$ & $26(43.33)$ & & \\
\hline \multicolumn{5}{|l|}{ Diabetes } \\
\hline Yes & $40(58.82)$ & $24(40.00)$ & 4.518 & 0.034 \\
\hline No & $28(47.18)$ & $36(60.00)$ & & \\
\hline \multicolumn{5}{|l|}{ Hypertension } \\
\hline Yes & $41(60.29)$ & $22(36.67)$ & 7.120 & 0.008 \\
\hline No & $27(39.71)$ & $38(63.33)$ & & \\
\hline \multicolumn{5}{|c|}{ Hyperlipidemia } \\
\hline Yes & $38(55.88)$ & $19(31.67)$ & 7.567 & 0.006 \\
\hline No & $30(41.12)$ & $41(61.33)$ & & \\
\hline \multicolumn{5}{|c|}{ Place of residence } \\
\hline Urban area & $29(42.65)$ & $23(38.33)$ & 0.246 & 0.621 \\
\hline Rural area & $39(57.35)$ & $37(61.67)$ & & \\
\hline
\end{tabular}

$2 \mathrm{~min}$, the washing solution was shaken off, and each well was patted dry with absorbent paper. After 6 repeats of the above steps, each well was added with $50 \mu 1$ of substrate A and $50 \mu 1$ of substrate $\mathrm{B}$, and incubated at $37^{\circ} \mathrm{C}$ in the dark for $10 \mathrm{~min}$. Each well was added with $50 \mu 1$ of stop solution, and within 15 min after the addition, the optical density (OD) of each well was measured at $450 \mathrm{~nm}$ wavelength and the concentration was calculated.

Observation indexes. Primary observation indexes: The serum chemokine CXCL13 and IL-6 in group A were compared with those in group $\mathrm{B}$, and receiver operating characteristic (ROC) curves of serum chemokine CXCL13 and IL-6 in predicting lower limb vein thrombus after hip replacement were drawn.

Secondary observation indexes: The Pearson's correlation coefficient was used to analyze the correlation between serum chemokine CXCL13 and serum IL-6, and logistic regression analysis was employed to analyze risk factors affecting hip replacement.

Statistical analysis. In the present study, the collected data were analyzed statistically using SPSS20.0 software package, 
Table II. Predictive value of CXCL13 and IL-6 levels in lower limb vein thrombus after hip replacement.

\begin{tabular}{lcccccr}
\hline Index & AUC & $95 \%$ CI & Specificity $\%$ & Sensitivity $\%$ & Youden index $\%$ & Cut-off \\
\hline CXCL13 & 0.763 & $67.67 \sim 89.22$ & 61.76 & 80.00 & 43.24 & 55.491 \\
IL-6 & 0.710 & $53.31 \sim 78.31$ & 70.59 & 66.67 & 38.73 & 86.557 \\
\hline
\end{tabular}

AUC, area under the curve; cut-off, cut-off point; CXCL13, serum chemokine CXC ligand 13; IL-6, interleukin-6.

A
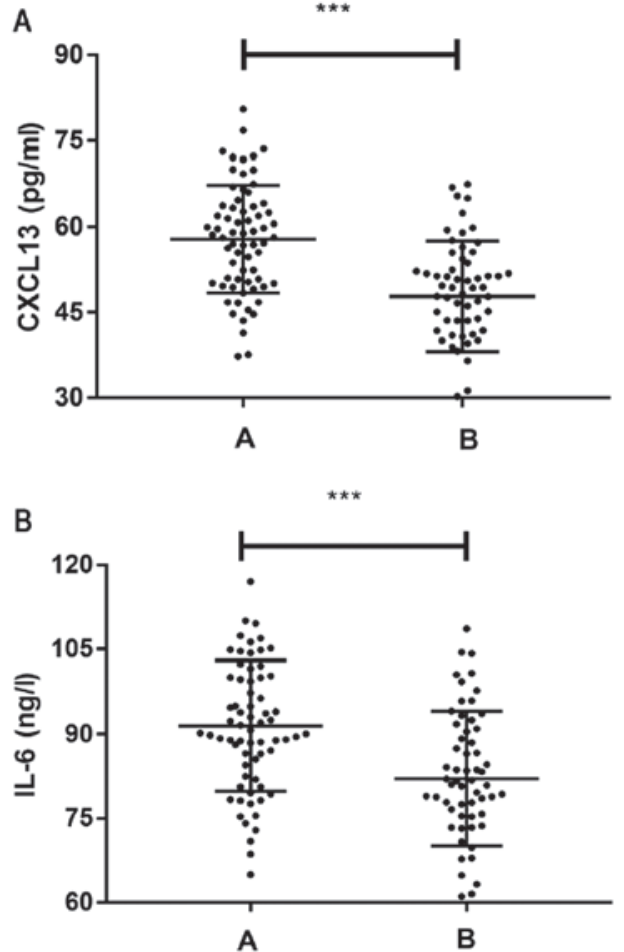

Figure 1. Expression of CXCL13 and IL-6 in patients. (A) Expression of CXCL13 in patients in Group A and Group B. (B) Expression of IL-6 in patients in Group A and Group B. ${ }^{* * *} \mathrm{P}<0.001$.

and visualized into required figures using GraphPad 7 software package. General data of the patients were analyzed using the chi-square test, and biological indexes were analyzed using the $\mathrm{t}$-test. The data in normal distribution were expressed by the mean \pm standard deviation (SD), and comparison between groups was carried out by the independent-samples t-test. Data not in normal distribution were expressed by the quartile [Mean (P25-P75)], and ROC curves of serum chemokine CXCL13 and IL-6 for predicting lower limb vein thrombus after hip replacement were drawn. $\mathrm{P}<0.05$ indicates a statistically significant difference. Pearson's correlation coefficient was used to analyze the correlation between serum chemokine CXCL13 and IL-6, and logistic regression analysis to analyze the risk factors affecting the efficacy of hip replacement.

\section{Results}

General data of patients. There were significant differences between the two groups in age, body mass index (BMI), diabetes history, hypertension history, and hyperlipidemia history (all $\mathrm{P}<0.05$ ), but there were no significant differences

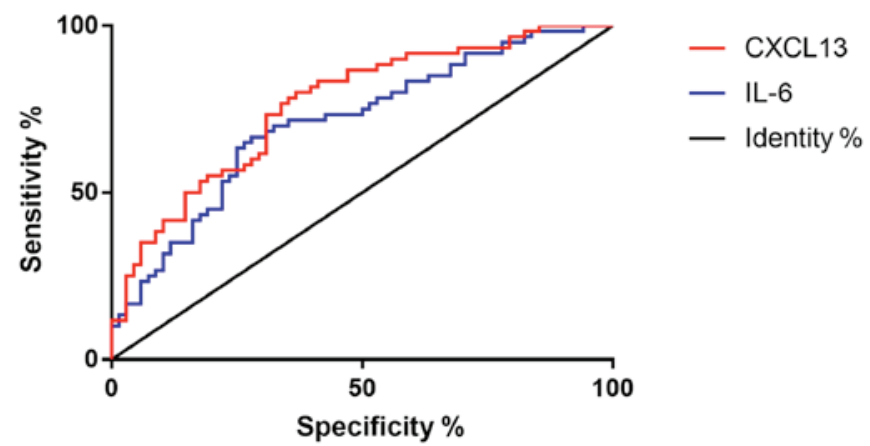

Figure 2. ROC curves of CXCL13 and IL-6 levels in predicting lower limb vein thrombus after hip replacement. AUC of CXCL13 was 0.763 , and that of IL-6 was 0.710. CXCL13, serum chemokine CXC ligand 13; IL-6, interleukin-6; AUC, area under the curve; ROC, receiver operating characteristic.

between them in sex, smoking history, drinking history, and place of residence (all $\mathrm{P}>0.05$ ) (Table I).

Expression of CXCL13 and IL-6 in group A and B. Comparison between group A and B in CXCL13 and IL-6 levels revealed that group A showed significantly higher CXCL13 and IL-6 levels than B (both $\mathrm{P}<0.05)$ (Fig. 1).

ROC curves of CXCL13 and IL-6 levels in predicting lower limb vein thrombus after surgery. The levels of CXCL13 and IL-6 in group A were compared with those in group B, and ROC curves of CXCL13 and IL-6 levels in predicting lower limb vein thrombus after hip replacement were drawn (Fig. 2 and Table II).

Analysis on the correlation between serum CXCL13 and serum $I L-6$ in the patients. Based on the comparison between the two groups in CXCL13 and IL-6 levels, the Pearson's correlation coefficient was employed to analyze the correlation between the two factors, and it was found that they were positively correlated (Fig. 3).

Risk factors affecting efficacy. The results in Table I show that the age of the patients, BMI, diabetes history, hyperlipidemia history, and hypertension history were independent factors affecting hip replacement, and our study results revealed that the CXCL13 and IL-6 levels in the patients were also independent factors affecting hip replacement. Therefore, we adopted the logistic regression analysis to analyze the risk factors for hip replacement by taking age, BMI, diabetes history, hyperlipidemia history, hypertension history, CXCL13 level and IL-6 level as covariates. Assignment was carried out on 
Table III. Assignments.

\begin{tabular}{lc}
\hline Factors & Assignment \\
\hline Age $($ years $)$ & $<30=0, \geq 30=1$ \\
BMI $\left(\mathrm{kg} / \mathrm{m}^{2}\right)$ & $<22=0, \geq 22=1$ \\
CXC13 $(\mathrm{pg} / \mathrm{ml})$ & $<50.92=0, \geq 50.92=1$ \\
IL-6 $(\mathrm{ng} / \mathrm{l})$ & $<88.15=0, \geq 88.15=1$ \\
Diabetes history & Yes $=0, \mathrm{No}=1$ \\
Hyperlipidemia history & $\mathrm{Yes}=0, \mathrm{No}=1$ \\
Hypertension history & $\mathrm{Yes}=0, \mathrm{No}=1$ \\
Lower limb vein thrombus & $\mathrm{Yes}=0, \mathrm{No}=1$ \\
\hline
\end{tabular}

independent factors based on the original data (Table III). The final results showed that age, BMI, diabetes history, hyperlipidemia history, hypertension history, CXCL13 level, and IL-6 level were independent risk factors affecting the efficacy of hip replacement (all $\mathrm{P}<0.05)$ (Table IV).

\section{Discussion}

With the development of global aging, joint diseases such as various osteoarthritis, bone fractures, and femoral head necrosis become an important health problem $(17,18)$, and with the improvement of medical level, hip replacement has become a reliable treatment (19). Hip replacement is a mature and reliable treatment method, which fixes artificial prosthesis, including femoral and acetabular parts, on normal bones with bone cement and screws to replace diseased joints and rebuild the normal function of hip joint of patients. It has the following advantages: Contributing to good joint motion and early off-bed activity, and reducing long-term bedridden complications of elderly patients $(20,21)$. However, it has the characteristics of high difficulty and high risk. Lower limb vein thrombus after hip replacement is the most common complication affecting its efficacy and prognosis, and is a crucial factor. It may cause partial or complete loss of limb function and disability, and may even cause pulmonary embolism and endanger life under serious situations (22). Predicting the risk of lower limb vein thrombus after hip replacement in advance and taking appropriate protective measures in time are the main methods to reduce lower limb vein thrombus. Finding sensitive biological indexes is also the focus of clinical research $(23,24)$.

In this study, comparison between group A and B in serum CXCL13 and IL-6 levels revealed that group A showed significantly higher CXCL13 and IL-6 levels than group B (both $\mathrm{P}<0.001$ ). Some studies have concluded that serum CXCL13 and IL-6 are important pro-inflammatory factors, and involved in occurrence and development of inflammatory reactions $(10,13)$ and the development of inflammation often induces thrombus, which is one of the mechanisms of thrombosis (14). Wang et al (25) compared the levels of inflammation markers and coagulation factors in 59 patients with lower limb vein thrombus and those in 26 patients without it, finding that inflammatory reaction and coagulation factors interact with each other to promote coagulation.

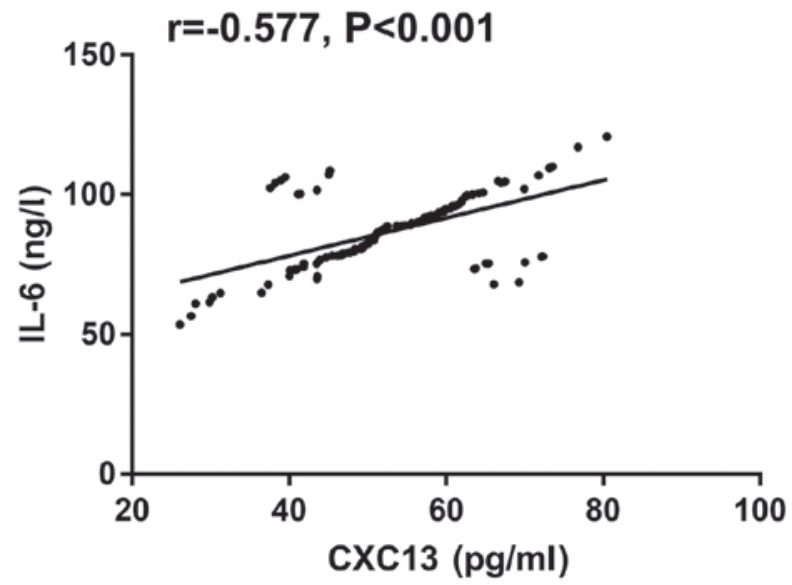

Figure 3. Pearson's correlation coefficient analysis of serum CXCL13 and serum IL-6 in patients, $r=-0.577, \mathrm{P}<0.001$. CXCL13, serum chemokine $\mathrm{CXC}$ ligand 13; IL-6, interleukin-6.

The development of inflammatory activities often leads to the increase of coagulation factors. Therefore, inflammatory reaction is closely related to lower limb venous thrombosis and is one of its mechanisms. Based on the present study, we deduced that inflammatory response was one of the mechanisms involved in lower limb vein thrombus after hip replacement, so patients with lower limb vein thrombus after hip replacement would show significantly higher serum CXCL13 and IL-6 levels than those without lower limb vein thrombus after surgery.

The sensitivity and specificity were also compared of separate serum CXCL13 level or serum IL-6 level and those of combined serum CXCL13 level and serum IL-6 level in diagnosing lower limb vein thrombus after hip replacement, finding that serum CXCL13 and IL-6 levels have certain value in diagnosis after hip replacement. Serum CXCL13 and IL-6 are important pro-inflammatory factors $(10,13)$ and inflammatory activity is one of the important mechanisms of thrombosis (14). Based on this study, it was concluded that serum CXCL13 and IL-6 can be used as biological indicators to diagnose lower limb vein thrombus after hip replacement. Logistic regression analysis revealed that patients' age, BMI, diabetes history, hyperlipidemia history, hypertension history, CXCL13 level, and IL-6 level were independent risk factors affecting the efficacy of hip replacement. Therefore, for patients who are older or have a relatively high BMI index, diabetes history, hyperlipidemia history or hypertension history, more protective measures should be given to prevent lower limb vein thrombus after surgery. For example, asking the patients turn over regularly and take appropriate activities, observing their lower limb status, and strengthening nursing work. Moreover, corresponding protective measures could be taken in advance to prevent lower limb vein thrombus based on detected serum CXCL13 and IL-6 levels in patients after surgery.

In conclusion, the levels of CXCL13 and IL-6 in the serum of patients with lower limb venous thrombosis after hip replacement significantly increased, which can be used as biological indicators for early prediction of lower limb venous thrombosis after hip replacement. 
Table IV. Risk factors for efficacy of hip replacement.

$95 \%$ CI of $\operatorname{EXP}(\mathrm{B})$

\begin{tabular}{lllllrrr} 
Factors & B & SD & Wals & Sig. & Exp (B) & Lower limit & Upper limit \\
\hline Age & 2.122 & 0.515 & 16.961 & 0.009 & 8.345 & 3.04 & 22.904 \\
BMI & 1.65 & 0.503 & 10.765 & 0.015 & 5.207 & 1.943 & 13.951 \\
CXC13 & 1.353 & 0.485 & 7.785 & 0.005 & 3.867 & 0.496 & 0.928 \\
IL-6 & 0.848 & 0.471 & 3.245 & 0.008 & 2.335 & 0.479 & 3.121 \\
Diabetes history & 0.201 & 0.478 & 0.177 & 0.032 & 1.223 & 5.926 \\
Hypertension history & 0.804 & 0.498 & 2.605 & 0.034 & 2.233 & 0.672 \\
Hyperlipidemia history & 0.56 & 0.489 & 1.313 & 0.025 & 1.751 & 4.562 \\
\hline
\end{tabular}

B, constant term; SD, standard deviation; Wals, chi-square value; sig, P-value; Exp (B), dominance ratio; 95\% CI of EXP (B), 95\% confidence interval of dominance ratio; BMI, body mass index; CXCL13, serum chemokine CXC ligand 13; IL-6, interleukin-6.

\section{Acknowledgements}

Not applicable.

\section{Funding}

No funding was received.

\section{Availability of data and materials}

The datasets used and/or analyzed during the current study are available from the corresponding author on reasonable request.

\section{Authors' contributions}

ZG wrote the manuscript and designed the study. ZG approved the final manuscript.

\section{Ethics approval and consent to participate}

The study was approved by the Ethics Committee of The First Affiliated Hospital of Guizhou University of Traditional Chinese Medicine (Guiyang, China). Patients who participated in this study, signed the informed consent and had complete clinical data.

\section{Patient consent for publication}

Not applicable.

\section{Conflict of interest}

The author declares that there are no competing interests.

\section{References}

1. Learmonth ID, Young $\mathrm{C}$ and Rorabeck $\mathrm{C}$ : The operation of the century: Total hip replacement. Lancet 370: 1508-1519, 2007

2. Galia CR, Diesel CV, Guimarães MR and Ribeiro TA: Total hip arthroplasty: A still evolving technique. Rev Bras Ortop 52: 521-527, 2017.

3. Zagra L: Advances in hip arthroplasty surgery: What is justified? EFORT Open Rev 2: 171-178, 2017.
4. Pivec R, Johnson AJ, Mears SC and Mont MA: Hip arthroplasty. Lancet 380: 1768-1777, 2012.

5. Knight SR, Aujla R and Biswas SP: Total Hip Arthroplasty - over 100 years of operative history. Orthop Rev (Pavia) 3: e16, 2011. doi: 10.4081/or.2011.e16.

6. Rogers BA, Garbedian S, Kuchinad RA, Backstein D, Safir O and Gross AE: Total hip arthroplasty for adult hip dysplasia. J Bone Joint Surg Am 94: 1809-1821, 2012.

7. Nam D, Nunley RM, Johnson SR, Keeney JA, Clohisy JC and Barrack RL: The effectiveness of a risk stratification protocol for thromboembolism prophylaxis after hip and knee arthroplasty. J Arthroplasty 31: 1299-1306, 2016.

8. Fukushima K, Takahira N, Uchiyama K, Moriya M, Minato T and Takaso M: The incidence of deep vein thrombosis (DVT) during hip arthroscopic surgery. Arch Orthop Trauma Surg 136: 1431-1435, 2016.

9. Han BK, Kuzin I, Gaughan JP, Olsen NJ and Bottaro A: Baseline CXCL10 and CXCL13 levels are predictive biomarkers for tumor necrosis factor inhibitor therapy in patients with moderate to severe rheumatoid arthritis: A pilot, prospective study. Arthritis Res Ther 18: 93, 2016.

10. Allam SI, Sallam RA, Elghannam DM and El-Ghaweet AI: Clinical significance of serum B cell chemokine (CXCL13) in early rheumatoid arthritis patients. Egypt Rheumatol 41: 11-14, 2019.

11. Irani DN: Regulated production of CXCL13 within the central nervous system. J Clin Cell Immunol 7: 7, 2016.

12. Sapan HB, Paturusi I, Jusuf I, Patellongi I, Massi MN, Pusponegoro AD, Arief SK, Labeda I, Islam AA, Rendy L, et al: Pattern of cytokine (IL-6 and IL-10) level as inflammation and anti-inflammation mediator of multiple organ dysfunction syndrome (MODS) in polytrauma. Int J Burns Trauma 6: 37-43, 2016.

13. Rose-John S: The soluble interleukin 6 receptor: Advanced therapeutic options in inflammation. Clin Pharmacol Ther 102: 591-598, 2017.

14. Iba T and Levy JH: Inflammation and thrombosis: Roles of neutrophils, platelets and endothelial cells and their interactions in thrombus formation during sepsis. J Thromb Haemost 16: 231-241, 2018.

15. Lankeit $\mathrm{M}$ and Held $\mathrm{M}$ : Incidence of venous thromboembolism in COPD: Linking inflammation and thrombosis? Eur Respir J 47: 369-373, 2016.

16. Wang J, Yang L, Li Q, Wu Z, Sun Y, Zou Q, Li X, Xu Z and Ye C: Construction of an adherence rating scale for exercise therapy for patients with knee osteoarthritis. BMC Musculoskelet Disord 19: 263, 2018.

17. Rahmati M, Nalesso G, Mobasheri A and Mozafari M: Aging and osteoarthritis: Central role of the extracellular matrix. Ageing Res Rev 40: 20-30, 2017.

18. Li YS, Xiao WF and Luo W: Cellular aging towards osteoarthritis. Mech Ageing Dev 162: 80-84, 2017.

19. Gademan MG, Hofstede SN, Vliet Vlieland TP, Nelissen RG and Marang-van de Mheen PJ: Indication criteria for total hip or knee arthroplasty in osteoarthritis: A state-of-the-science overview. BMC Musculoskelet Disord 17: 463, 2016. 
20. Liu XW, Zi Y, Xiang LB and Wang Y: Total hip arthroplasty: A review of advances, advantages and limitations. Int J Clin Exp Med 8: 27-36, 2015.

21. Burnett RSJ and Abos D: Total hip arthroplasty: Techniques and results. BC Med J 52:455-464, 2010.

22. Jameson SS, Bottle A, Malviya A, Muller SD and Reed MR: The impact of national guidelines for the prophylaxis of venous thromboembolism on the complications of arthroplasty of the lower limb. J Bone Joint Surg Br 92: 123-129, 2010.

23. Shimoyama Y, Sawai T, Tatsumi S, Nakahira J, Oka M, Nakajima M, Jotoku T and Minami T: Perioperative risk factors for deep vein thrombosis after total hip arthroplasty or total knee arthroplasty. J Clin Anesth 24: 531-536, 2012.
24. Fisher WD: Impact of venous thromboembolism on clinical management and therapy after hip and knee arthroplasty. Can J Surg 54: 344-351, 2011

25. Wang MF, Yang LH, Yang XL, Zhang RJ, Hou LH and Liu XE: Correlation of inflammatory marker and coagulation factors with deep vein thrombosis. Zhongguo Shi Yan Xue Ye Xue Za Zhi 18: 753-756, 2010 (In Chinese) 